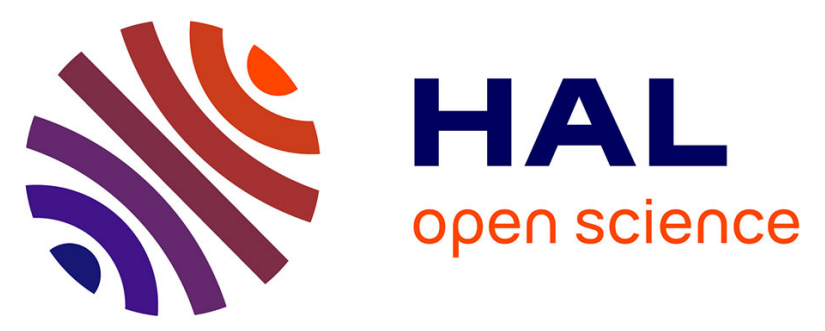

\title{
A model to describe the cyclic anisotropic mechanical behavior of short fiber-reinforced thermoplastics
}

Libor Navratil, Louis Leveuf, V. Le Saux, Yann Marco, J. Olhagaray, S. Leclercq, Sylvain Moyne, Matthieu Le Saux

\section{- To cite this version:}

Libor Navratil, Louis Leveuf, V. Le Saux, Yann Marco, J. Olhagaray, et al.. A model to describe the cyclic anisotropic mechanical behavior of short fiber-reinforced thermoplastics. 24e Congrès Français de Mécanique, Aug 2019, Brest, France. hal-02493873

\section{HAL Id: hal-02493873 \\ https://hal-ensta-bretagne.archives-ouvertes.fr/hal-02493873}

Submitted on 28 Feb 2020

HAL is a multi-disciplinary open access archive for the deposit and dissemination of scientific research documents, whether they are published or not. The documents may come from teaching and research institutions in France or abroad, or from public or private research centers.
L'archive ouverte pluridisciplinaire HAL, est destinée au dépôt et à la diffusion de documents scientifiques de niveau recherche, publiés ou non, émanant des établissements d'enseignement et de recherche français ou étrangers, des laboratoires publics ou privés. 


\title{
A model to describe the cyclic anisotropic mechanical behavior of short fiber-reinforced thermoplastics
}

\author{
L. NAVRATIL ${ }^{a}$, L. LEVEUF ${ }^{b}$, V. LE SAUX ${ }^{a}$, Y. MARCO ${ }^{a}$, J. \\ OLHAGARAY ${ }^{c}$, S. LECLERCQ ${ }^{d}$, S. MOYNE ${ }^{a}$, M. LE SAUX ${ }^{a}$
}

a. ENSTA Bretagne, UMR CNRS 6027, IRDL, F-29200 Brest, France, libor.navratil@ensta-bretagne.org, vincent.le_saux@ensta-bretagne.fr, yann.marco@ensta-bretagne.fr, sylvain.moyne@ensta-bretagne.fr, matthieu.le_saux@ensta-bretagne.fr b. formely at ENSTA Bretagne, UMR CNRS 6027, IRDL, F-29200 Brest, France, louis.leveuf@ensta-bretagne.org c. Safran Composites, F-91760, Itteville, France, jerome.olhagaray@ safrangroup.com d. Safran Landing systems, F-78140, Vélizy, France, sylvain.leclercq@ safrangroup.com

\begin{abstract}
:
Due to the injection molding process, short fiber-reinforced thermoplastic composites show a complex fiber orientation distribution and, as a consequence, an overall anisotropic mechanical behavior. The monotonic and cyclic mechanical behavior of PolyEtherEtherKetone thermoplastic reinforced with 30wt.\% of short carbon fibers was characterized through a series of tests generating various complex loading histories performed at room temperature on samples with various homogeneous and heterogeneous fiber orientation distributions. A three-dimensional model relying on a thermodynamic framework was then developed to represent the anisotropic mechanical behavior of the material, including elastic, viscoelastic and plastic phenomena. The model was implemented into a finite element code to be able to simulate the response of complex parts with a heterogeneous fiber orientation distribution subjected to a heterogeneous loading. Model parameters were identified by applying a robust and original approach relying on a limited number of relevant experiments. The prediction capability of the model was demonstrated by simulating several types of tests not used for the identification, covering a wide range of monotonic and cyclic, homogeneous and heterogeneous, loading conditions, for various simple and complex fiber orientation distributions. In particular, the model is shown to be able to predict the energy dissipated in the material when subjected to cyclic loading.
\end{abstract}

\section{Keywords : short fiber-reinforced thermoplastic, anisotropic non-linear me- chanical behavior, model, fiber orientation}

\section{Introduction}

Short fiber-reinforced thermoplastics (SFRT) are increasingly used in the industry. They can be subjected to complex cyclic loading during their service life. As a consequence, their resistance to fatigue is 


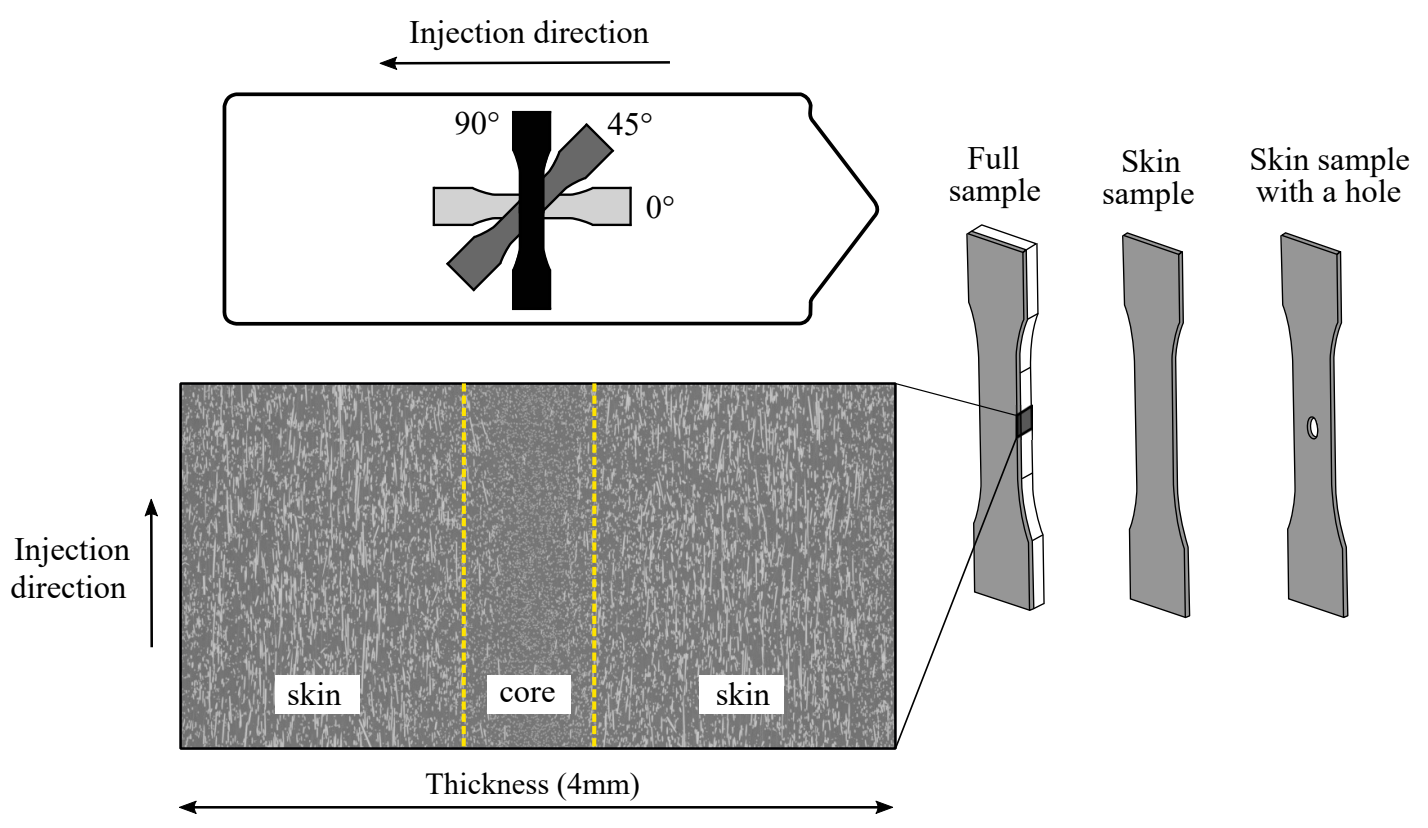

FiguRE 1 - Microstructure of the tested material and specimens used for this study.

an important issue. The design against fatigue failure often makes use, on the one hand, of a model describing the cyclic thermomechanical behavior of the material and, on the other hand, of a fatigue criterion. To establish a predictive approach against fatigue, it is thus necessary to have an accurate description of the cyclic mechanical behavior of the material. In particular, it is useful to be able to predict dissipation during cyclic loading since it can be related to fatigue lifetime, through energy-based criterion for example [1, 2]. Due to the injection molding process, these materials show a complex heterogeneous microstructure, with heterogeneous distribution of fiber orientation in particular. The behavior of the composite material naturally depends on the properties of both the matrix and the fibers as well as on the content, the distribution and the orientation of the fibers. This paper presents a model describing the cyclic mechanical behavior of SFRT materials, in particular of PolyEtherEtherKetone (PEEK) thermoplastic reinforced with $30 \mathrm{wt} . \%$ of short carbon fibers, meant for an aeronautical application. The objective is to propose a robust and efficient model that can easily be used in an industrial context to simulate complex parts. More details on the model and the experimental database used for its identification and validation are given in [9]. The model is an anisotropic extension of the model proposed in [3], describing the material behavior for one specific single average fiber orientation.

\section{Material and experiments}

\subsection{Material}

In order to establish the experimental database required to identify and validate the model, a series of tests generating various complex loading histories was performed at room temperature on samples with various homogeneous and heterogeneous fiber orientation distributions. The experiments were made on samples cut from $4 \mathrm{~mm}$-thick injection molded plates of PEEK reinforced with 30wt. \% of short carbon fibers. Diameter and length of fibers are respectively smaller than $10 \mu \mathrm{m}$ and $200 \mu \mathrm{m}$ in average. As shown in Fig. 1. the material exhibits a strong through-thickness gradient of fiber orientation. Specimens 
with the geometries shown in Fig. 11(50 mm-long and $10 \mathrm{~mm}$-wide gauge part) were taken from the injection molded plates, for mechanical testing :

- full-thickness $(4 \mathrm{~mm})$ specimens called full samples hereinafter, with a through-thickness fiber orientation gradient;

- 1 mm-thick specimens called skin samples obtained from the skin of full samples in order to get a "homogeneous" microstructure;

— skin samples with a central $3 \mathrm{~mm}$-diameter hole.

To characterize the anisotropic behavior of the material, the samples were milled out so that their gauge length and as a consequence the main loading direction during the tests were oriented at 0,45 or $90^{\circ}$ from the injection direction. The skin samples allow to characterize the behavior of the material with a "homogeneous" well-controlled microstructure (distribution of fibers), free of the "skin-core"-induced structural effects of full samples. The results of the tests performed on these samples are used to identify some of the model parameters. The results obtained on full samples, showing a heterogeneous distribution of fibers, are exploited to identify the model parameters relative to anisotropy in particular. The tests performed on skin samples with a hole are used to generate data aiming at validating the model in the case of a complex heterogeneous local loading.

The orientation of fibers is expected to have a first-order effect on the mechanical behavior of the material. The effects of other sources of heterogeneity are neglected. As proposed in [4] for example, the microstructure is characterized by the distribution of fibers orientations, described by a second-order tensor $\underline{\boldsymbol{a}}^{\psi}$ and a fourth-order tensor $\underline{\underline{\boldsymbol{A}}}^{\psi} \cdot \underline{\boldsymbol{a}}^{\psi}$ was determined from 2D optical micrographs by following the procedure described in [5]. $\underline{\underline{A}}^{\psi}$ is estimated from $\underline{\boldsymbol{a}}^{\psi}$ using the orthotropic fitted closure equation proposed by Cintra and Tucker [6].

\subsection{Experiments}

Several types of tensile tests were performed to characterize the mechanical behavior of the material and to build an appropriate database, including a large set of loading conditions, to develop and validate the model describing this behavior. The tests were performed at room temperature (around $20^{\circ} \mathrm{C}$ ) and room humidity (approximately 50\%). As already proposed in [3] and [7], the strategy relies on limited in number but carefully chosen experiments, with complex loading histories to catch the deformation mechanisms and to determine the ingredients to be included in the model. The following tests were performed :

- Monotonic tensile tests at various loading rates from 0.06 to $60 \mathrm{kN} / \mathrm{min}$.

- Cyclic tensile creep-recovery tests consisting in creep steps at several stress levels separated with a recovery period at zero stress, with increasing then decreasing stress levels applied during the creep steps. Creep and recovery times between a few minutes and one hour were applied to observe mean and long-term viscous effects. Each creep step was repeated two times in order to be able to separate viscoelastic and (visco)plastic effects. Some cyclic creep recovery tests were also performed with an alternation of tensile and compression creep steps with the same stress amplitude.

- Anhysteretic tests consisting in loading-unloading tension or compression tests with creep steps (duration from 5 to a few tens of minutes) at various stress levels during loading and unloading sequences.

- Tension-relaxation-recovery tests consisting in applying successively tension, relaxation (at zero strain) and recovery (at zero stress) steps. The tests were performed with three strain levels. 
- Cyclic tests consisting in applying a load-controlled sinusoidal cyclic loading. Several stress levels with a zero stress ratio were applied, with frequencies of 1,2 or $5 \mathrm{~Hz}$.

- Heat build-up tests consisting in applying successive sequences of load-controlled sinusoidal cyclic loading at 1 or $2 \mathrm{~Hz}$ with increasing stress levels. The sample is left to cool during a few minutes at zero stress between each loading sequence so that they get back to thermal equilibrium with ambient. Temperature variation at the sample surface was measured during heat build-up tests by using an infrared camera. The cyclic dissipation was evaluated from the measured evolution of temperature using the methodology described in [1, 2, 8].

During these tests, the in-plane displacement fields at the sample surface were measured by 2D digital image correlation. To provide the contrast needed to correlate images, a random speckle patterns was applied by black, white and gray paints sprays. The mean strain within the specimen gauge length was obtained by spatial averaging of the strain field.

The results of the tests, already partly presented in [3], are not detailed in this paper. Only the main conclusions are given. More details can be found in [9]. The material exhibits anisotropic elasticity, non-permanent non-linear viscous effects associated with plastic hardening, probably of kinematic kind, and permanent strains even after a long recovery period which could be described by a timeindependent anisotropic plasticity. Viscoelasticity appears to be predominant compared to viscoplasticity. Manifestation of damage appears lately during cyclic loading. As a consequence, it is not necessary to take damage into account to model a stabilized state under cyclic loading. These conclusions apply whatever the orientation of the sample with respect to the injection and loading directions.

\section{Modeling}

\subsection{General formulation}

The model relies on the hypothesis that the cyclic mechanical behavior and damage in fatigue can be decoupled so that the fatigue life can be determined from the mechanical behavior measured in stationary regime [10]. The model is written within the framework of generalized standard materials, as defined in [11]. The mechanical state of the material is defined by the second-order strain tensor $\underline{\varepsilon}$ associated to the stress tensor $\underline{\sigma}$, the viscoelastic strain $\underline{\varepsilon}_{\mathrm{v}}$ associated to the thermodynamic force $\underline{\mathcal{A}}_{\mathrm{v}}$, the plastic strain $\underline{\varepsilon}_{\mathrm{p}}$ associated to the thermodynamic force $\underline{\mathcal{A}}_{\mathrm{p}}$ and the hardening variable $\underline{\alpha}$ associated to the back-stress (center of the yield surface) $\underline{\boldsymbol{X}}$. Using the infinitesimal strain theory, total strain $\underline{\varepsilon}$ is additively decomposed in elastic, viscoelastic and plastic components, noted $\underline{\varepsilon}_{\mathrm{e}}, \underline{\varepsilon}_{\mathrm{v}}$ and $\underline{\varepsilon}_{\mathrm{p}}$, respectively :

$$
\underline{\varepsilon}=\underline{\varepsilon}_{\mathrm{e}}+\underline{\varepsilon}_{\mathrm{v}}+\underline{\varepsilon}_{\mathrm{p}}
$$

The thermodynamic forces are calculated as follows :

$$
\begin{gathered}
\underline{\boldsymbol{\sigma}}=\rho \frac{\partial \psi}{\partial \underline{\varepsilon}}=\underline{\underline{\boldsymbol{C}}}_{\mathrm{e}}:\left(\underline{\varepsilon}-\underline{\boldsymbol{\varepsilon}}_{\mathrm{v}}-\underline{\boldsymbol{\varepsilon}}_{\mathrm{p}}\right) \\
\underline{\mathcal{A}}_{\mathrm{v}}=-\rho \frac{\partial \psi}{\partial \underline{\boldsymbol{\varepsilon}}_{\mathrm{v}}}=-\left(\underline{\boldsymbol{\sigma}}-\underline{\boldsymbol{C}}_{\mathrm{v}}(\underline{\boldsymbol{\alpha}}): \underline{\boldsymbol{\varepsilon}}_{\mathrm{v}}\right) \\
\underline{\mathcal{A}}_{\mathrm{p}}=-\rho \frac{\partial \psi}{\partial \underline{\varepsilon}_{\mathrm{p}}}=\underline{\boldsymbol{\sigma}}
\end{gathered}
$$




$$
\underline{\boldsymbol{X}}=\rho \frac{\partial \psi}{\partial \underline{\boldsymbol{\alpha}}}=\frac{2}{3} C \underline{\boldsymbol{\alpha}}+\frac{\partial}{\partial \underline{\boldsymbol{\alpha}}}\left(\frac{1}{2} \underline{\boldsymbol{\varepsilon}}_{\mathrm{v}}: \underline{\underline{C}}_{\mathrm{v}}(\underline{\boldsymbol{\alpha}}): \underline{\boldsymbol{\varepsilon}}_{\mathrm{v}}\right) \approx \frac{2}{3} C \underline{\boldsymbol{\alpha}}
$$

where $\rho$ is the density, $\underline{\underline{C}}$ is the fourth-order (instantaneous) elastic stiffness tensor and $\underline{\underline{C}}_{\mathrm{v}}$ is the viscoelastic stiffness tensor. As discussed hereinafter, $\underline{\underline{C}}_{\mathrm{v}}$ depends on $\underline{\boldsymbol{\alpha}}$. ":" denotes the double dot product (double contraction) of tensors. $C$ is a parameter depending on the material. The simplification in Eq. 5] is justified in [3]. The evolution laws of state variables, deduced from the generalized normality rule, are expressed as follows :

$$
\begin{gathered}
\dot{\varepsilon}_{\mathrm{v}}=-\frac{1}{\eta\left(\underline{\dot{\varepsilon}}_{\mathrm{v}}\right)} \underline{\mathcal{A}}_{\mathrm{v}}=\frac{1}{\eta\left(\underline{\dot{\varepsilon}}_{\mathrm{v}}\right)}\left(\underline{\boldsymbol{\sigma}}-\underline{\boldsymbol{C}}_{\mathrm{v}}(\underline{\boldsymbol{\alpha}}): \underline{\boldsymbol{\varepsilon}}_{\mathrm{v}}\right) \\
\dot{\boldsymbol{\varepsilon}}_{\mathrm{p}}=\dot{p} \underline{\boldsymbol{n}} \\
\underline{\dot{\boldsymbol{\alpha}}}=\dot{p} \underline{\boldsymbol{n}}-\frac{3}{2} \frac{\gamma}{C} \dot{p} \underline{\boldsymbol{X}}
\end{gathered}
$$

$\dot{p}$ is the norm of the plastic strain rate [17]:

$$
\dot{p}=\sqrt{\underline{\dot{\varepsilon}}_{\mathrm{p}}: \underline{\underline{H}}^{-1}: \underline{\dot{\varepsilon}}_{\mathrm{p}}}
$$

$\underline{\boldsymbol{n}}$ is the flow direction :

$$
\underline{\boldsymbol{n}}=\frac{\partial f_{\mathrm{p}}}{\partial \underline{\boldsymbol{\sigma}}}=\frac{\underline{\underline{\boldsymbol{H}}}:(\underline{\boldsymbol{\sigma}}-\underline{\boldsymbol{X}})}{\mathcal{J}_{\mathrm{p}}(\underline{\boldsymbol{\sigma}}-\underline{\boldsymbol{X}})}
$$

$f_{\mathrm{p}}(\underline{\boldsymbol{\sigma}}, \underline{\boldsymbol{X}})$ is the yield surface, defining the limit at which the material becomes plastic :

$$
f_{\mathrm{p}}(\underline{\boldsymbol{\sigma}}, \underline{\boldsymbol{X}})=\mathcal{J}_{\mathrm{p}}(\underline{\boldsymbol{\sigma}}-\underline{\boldsymbol{X}})-\sigma_{y}
$$

where $\sigma_{y}$ is the yield stress and $\mathcal{J}_{\mathrm{p}}$ is a norm of stresses :

$$
\mathcal{J}_{\mathrm{p}}(\underline{\boldsymbol{\sigma}}-\underline{\boldsymbol{X}})=\sqrt{(\underline{\boldsymbol{\sigma}}-\underline{\boldsymbol{X}}): \underline{\underline{\boldsymbol{H}}}:(\underline{\boldsymbol{\sigma}}-\underline{\boldsymbol{X}})}
$$

with $\underline{\underline{\boldsymbol{H}}}$ a symmetric fourth-order tensor describing plastic anisotropy. The Hill tensor is used here and $\dot{\varepsilon}_{\mathrm{p}}=\underline{\mathbf{0}}$ if $f_{\mathrm{p}}<0 . \gamma$ is a material parameter. In order to describe the evolution of the equivalent elastic stiffness within a wide stress range, during both loading and unloading stages, a dependency of $\underline{\underline{C}}_{\mathrm{v}}$ to kinematic hardening $\underline{\boldsymbol{X}}(\underline{\boldsymbol{\alpha}})$ is introduced :

$$
\underline{\underline{\boldsymbol{C}}}_{\mathrm{v}}=\underline{\underline{\boldsymbol{C}}}_{\mathrm{v} 0} \exp \left(-k_{\mathrm{v}} \mathcal{J}_{2}(\underline{\boldsymbol{X}})\right)
$$

where $k_{\mathrm{v}}$ is a parameter to be identified. $\mathcal{J}_{2}(\underline{\boldsymbol{X}})$ is the von Mises norm of the back stresses :

$$
\mathcal{J}_{2}(\underline{\boldsymbol{X}})=\sqrt{\frac{3}{2} \underline{\boldsymbol{X}}: \underline{\underline{\boldsymbol{K}}}: \underline{\boldsymbol{X}}}
$$

$\underline{\underline{K}}$ is the deviatoric projector of the fourth-order unit tensor $\underline{\underline{\boldsymbol{I}}}: \underline{\underline{\boldsymbol{K}}}=\underline{\underline{\boldsymbol{I}}}-\underline{\underline{\boldsymbol{J}}}$, where $\underline{\underline{\boldsymbol{J}}}=\frac{1}{3} \underline{\boldsymbol{I}} \otimes \underline{\boldsymbol{I}}$ is the spherical projector of $\underline{\underline{I}} . \underline{\underline{C}}_{\mathrm{v} 0}$ is the viscoelastic stiffness tensor when $\underline{\boldsymbol{X}}=\underline{\mathbf{0}}$. To catch the behavior of the material within a wide strain rate range and the effect of frequency in case of cyclic loading, the 
following non-linear viscosity law is used :

$$
\eta\left(\underline{\dot{\varepsilon}}_{\mathrm{v}}\right)=K\left(\sqrt{\frac{2}{3} \dot{\dot{\varepsilon}}_{\mathrm{v}}: \underline{\underline{\boldsymbol{K}}:} \underline{\dot{\varepsilon}}_{\mathrm{v}}}\right)^{n-1}
$$

$K$ and $n$ are material parameters.

\subsection{Elastic behavior}

As done in [4], elastic anisotropy is described with a two-step homogenization method. First, an equivalent unidirectional (UD) composite is determined. Second, in order to take the microstructure into account, a mean evaluation over the orientation distribution of the elementary UD composites is performed. Fibers and matrix are considered to have an isotropic and elastic behavior. According to the Mori-Tanaka model [12], for a dilute distribution of inclusions, the unidirectional (transversely isotropic) effective stiffness tensor is given by [13] :

$$
\underline{\underline{\boldsymbol{C}}}^{\mathrm{UD}}=\underline{\underline{\boldsymbol{C}}}_{m}+y_{f}\left(\underline{\underline{\boldsymbol{C}}}_{f}-\underline{\underline{\boldsymbol{C}}}_{m}\right):\left\langle\underline{\underline{\boldsymbol{L}}}_{f}^{\mathrm{UD}}\right\rangle
$$

where $y_{f}$ is the volume fraction of fibers. $\underline{\underline{C}}_{m}$ and $\underline{\underline{C}}_{f}$ are the elastic stiffness tensors of the matrix $(m)$ and the fibers $(f)$, respectively. They are deduced from the Hooke's law for isotropic materials, which can be expressed as a function of the Young's modulus $E_{n}$ and the Poisson's ratio $\nu_{n}$ (with $n=m, f$ ). In Eq. 16 . $\langle$.$\rangle is the averaging operator over fiber orientations. \underline{\underline{L}}_{f}^{\mathrm{UD}}$ is the localization tensor for fibers :

$$
\underline{\underline{\boldsymbol{L}}}_{f}^{\mathrm{UD}}=\underline{\underline{\boldsymbol{L}}}_{f}^{\mathrm{Esh}}:\left[\left(1-y_{f}\right) \underline{\underline{\boldsymbol{I}}}+y_{f} \underline{\underline{\boldsymbol{L}}}_{f}^{\mathrm{Esh}}\right]^{-1}
$$

$\underline{\underline{\boldsymbol{L}}}_{f}^{\mathrm{Esh}}$ is the localization tensor associated with fibers for a dilute medium where interaction effects can be neglected :

$$
\underline{\underline{\underline{L}}}_{f}^{\mathrm{Esh}}=\left[\underline{\underline{\boldsymbol{I}}}+\underline{\underline{\boldsymbol{S}}}: \underline{\underline{\boldsymbol{C}}}_{m}^{-1}:\left(\underline{\underline{\boldsymbol{C}}}_{f}-\underline{\underline{\boldsymbol{C}}}_{m}\right)\right]^{-1}
$$

where $\underline{\underline{S}}$ is the Eshelby tensor for an inclusion in a homogeneous infinite matrix. The fibers are assimilated to prolate spheroidal inclusions. The associated components of $\underline{\underline{\boldsymbol{S}}}$ can be found in [14]. They depend on the Poisson's ratio of the matrix $\nu_{m}$ and on the aspect ratio of the inclusion $t=l / d$, with $l$ the length of the inclusion and $d$ its diameter $\left(t_{f}>1\right.$ in the case of prolate spheroidal inclusions). As proposed in [4], the effective macroscopic elastic stiffness tensor is obtained by averaging the stiffnesses of the equivalent UD composites according to their orientation. It is expressed as a function of $\underline{a}^{\psi}$ and $\underline{\underline{A}}^{\psi}$ [4, 15] :

$$
\underline{\underline{\boldsymbol{C}}}_{\mathrm{e}}=K_{1} \underline{\underline{\boldsymbol{A}}}^{\psi}+K_{2}\left(\underline{\boldsymbol{a}}^{\psi} \otimes \underline{\boldsymbol{I}}+\underline{\boldsymbol{I}} \otimes \underline{\boldsymbol{a}}^{\psi}\right)+K_{3} \underline{\underline{\boldsymbol{D}}}\left(\underline{\boldsymbol{a}}^{\psi}, \underline{\boldsymbol{I}}\right)+3 K_{4} \underline{\underline{\boldsymbol{J}}}+2 K_{5} \underline{\underline{\boldsymbol{I}}}
$$

where $\otimes$ denotes the tensor product. $\underline{\boldsymbol{I}}$ is the second-order unit tensors. $\underline{\underline{\boldsymbol{D}}}$ is an operator applied to second-order tensors defined by $D_{i j k l}(\underline{\boldsymbol{a}}, \underline{\boldsymbol{b}})=a_{i k} b_{j l}+a_{i l} b_{j k}+a_{j l} b_{i k}+a_{j k} b_{i l}$. The constants $K_{i}$ with $i \in[1,5]$ are given in [16].

\subsection{Plastic behavior}

The mechanical behavior is stiffer along directions where many fibers are aligned but plasticity is more limited. Following [4], the Hill tensor, used to account for the influence of the fiber orientation distribution 
on the material plastic behavior, is expressed as follows in the orthotropy basis $\left(\boldsymbol{u}_{1}, \boldsymbol{u}_{2}, \boldsymbol{u}_{3}\right)$ :

$$
[\underline{\underline{\boldsymbol{H}}}]=\frac{3}{2}\left[\begin{array}{cccccc}
\left(1-a_{1}\right)^{k} & H_{1122} & H_{1133} & 0 & 0 & 0 \\
H_{1122} & \left(1-a_{2}\right)^{k} & H_{2233} & 0 & 0 & 0 \\
H_{1133} & H_{2233} & \left(1-a_{3}\right)^{k} & 0 & 0 & 0 \\
0 & 0 & 0 & (2 / 3)^{k-1} & 0 & 0 \\
0 & 0 & 0 & 0 & (2 / 3)^{k-1} & 0 \\
0 & 0 & 0 & 0 & 0 & (2 / 3)^{k-1}
\end{array}\right]_{\left(\boldsymbol{u}_{1}, \boldsymbol{u}_{2}, \boldsymbol{u}_{3}\right)}
$$

with

$$
\begin{aligned}
& H_{1122}=\frac{1}{2}\left(\left(1-a_{3}\right)^{k}-\left(1-a_{2}\right)^{k}-\left(1-a_{1}\right)^{k}\right) \\
& H_{1133}=\frac{1}{2}\left(\left(1-a_{2}\right)^{k}-\left(1-a_{3}\right)^{k}-\left(1-a_{1}\right)^{k}\right) \\
& H_{2233}=\frac{1}{2}\left(\left(1-a_{1}\right)^{k}-\left(1-a_{3}\right)^{k}-\left(1-a_{2}\right)^{k}\right)
\end{aligned}
$$

$k$ is a material parameter introduced to describe the sensitivity of the plastic behavior to the fiber orientation distribution [4].

\subsection{Viscoelastic behavior}

The results of the present study suggest that the material viscosity does not depend directly on the mean orientation of fibers. Nevertheless, a dependency exists as the viscoelastic stiffness depends on the kinematic hardening, which depends on the flow stress. The influence of the mean fiber orientation on the plastic flow stress seems to be sufficient to account for the dependency of the viscoelastic stiffness on the material microstructure. $\underline{\underline{C}}_{\mathrm{v} 0}$ in Eq. 13 is thus considered to be isotropic. It can be expressed as a function of a viscous modulus in the absence of back-stresses $E_{\mathrm{v} 0}$ and a viscous Poisson's ratio $\nu_{\mathrm{v}}$.

\subsection{Intrinsic dissipation}

Predicting the intrinsic dissipation during cyclic loading is one the main applications of the proposed model. Intrinsic dissipation can be expressed as follows [17] :

$$
\mathcal{D}=\underline{\sigma}: \underline{\dot{\varepsilon}}_{\mathrm{p}}-\underline{\boldsymbol{X}}: \underline{\dot{\alpha}}-\underline{\mathcal{A}}_{\mathrm{v}}: \underline{\dot{\varepsilon}}_{\mathrm{v}}
$$

In the case of cyclic loading, the average intrinsic dissipation during a cycle $N$ of duration $\mathcal{T}$ can then be estimated :

$$
\Delta_{\text {cyc }}=\frac{1}{\mathcal{T}} \int_{\text {cycle } N} \mathcal{D}(t) \mathrm{d} t
$$

\section{Identification of the model parameters and validation}

\subsection{Identification}

The model was implemented into the Abaqus ${ }^{\circledR}$ finite element code by using a UMAT (User MATerial) subroutine. The volume fraction $y_{f}$ and the orientation tensor $\underline{\boldsymbol{a}}^{\psi}$ of fibers are inputs of the model. For the present application, $y_{f}=0.23$ and $\underline{\boldsymbol{a}}^{\psi}$ was determined from microstructural observations. 

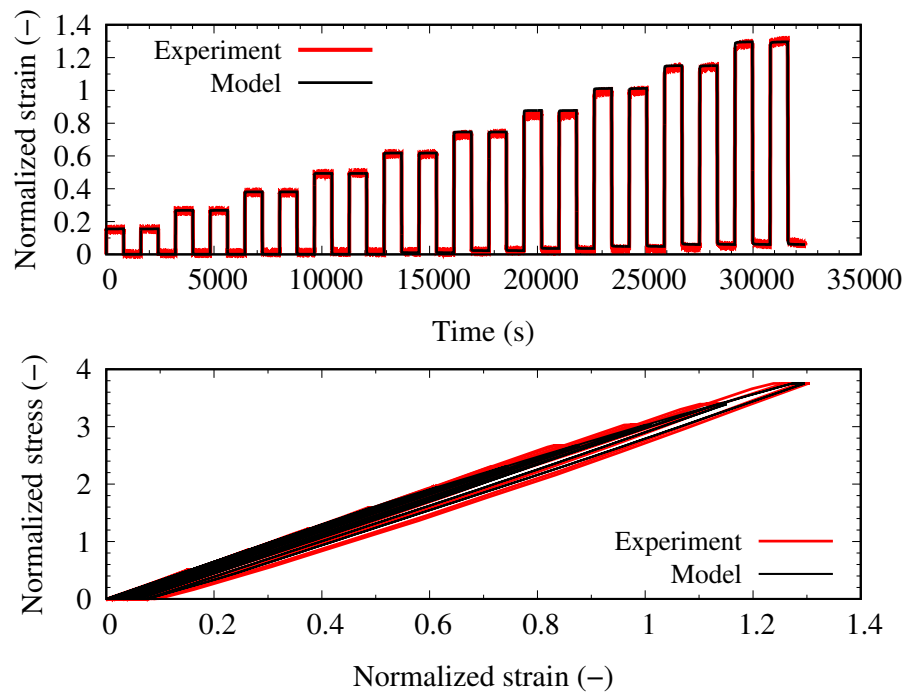

FIGURE 2 - Experimental and calculated results of a cyclic creep recovery test performed on a skin sample with its gauge length along the injection direction $\left(0^{\circ}\right.$ orientation).

The model includes 14 parameters to be identified. The macroscopic elastic modulus, $E_{\mathrm{v} 0}, \nu_{\mathrm{v}}, k_{\mathrm{v}}, K, n$, $\sigma_{y}, \gamma$ and $C$ were identified by using the procedure proposed in [3]. These parameters were tuned so that the model correctly predicts the response of cyclic creep recovery tests performed on skin samples with their gauge length along the injection direction $\left(0^{\circ}\right.$ orientation, fibers mainly oriented along the loading direction) on the one hand, perpendicular to the injection direction $\left(90^{\circ}\right.$ orientation, fibers mainly perpendicular to the loading direction) on the other hand. Only the values of the macroscopic elastic modulus and $\sigma_{y}$ really differ for $0^{\circ}$ orientation and $90^{\circ}$ orientation, confirming that the model formulation is relevant. The values of $E_{\mathrm{v} 0}, k_{\mathrm{v}}, K, n, \gamma$ and $C$ obtained for the $0^{\circ}$ orientation were then retained.

The identification of the parameters associated with the two-step homogenization method used to calculate the 3D elastic stiffness $\left(E_{f}, E_{m}, \nu_{f}, \nu_{m}\right.$ and $\left.t\right)$ and the parameter describing plastic anisotropy $(k)$ was based on the comparison of experimental and calculated results of tensile tests performed until failure on full, $3 \mathrm{~mm}$-thick, samples, with their gauge length oriented at 0,45 or $90^{\circ}$ from the injection direction. The fiber orientation distributions deduced from experimental observation of the sample cross-section were considered for the finite element simulations. It was considered that the observed microstructure is homogeneous along the sample volume. The values of $E_{m}, E_{f}$ and $t$ were tuned so that the difference between the measured and the calculated stress-strain responses in the elastic regime is minimized. Finally, the $k$ parameter was identified on the basis of the whole stress-strain responses. $\sigma_{y}$ was also readjusted. The values of the adjusted parameters can be found in [9].

\subsection{Validation}

For confidentiality purposes, the results are normalized by arbitrary values, similar for all the tests. The results of cyclic creep recovery tests performed on skin samples (homogeneous fiber orientation distribution), used to adjust some of the parameters, are naturally accurately reproduced by the model once tuned (Fig. 2). As shown in Figs. 3 and 4 it is also able to predict the results of cyclic and anhysteretic tests performed on skin samples, which were not used to identify model parameters. As shown in Fig. 5. the results of tensile tests performed until failure on full samples (with a through-thickness gradient of fiber orientation distribution), with several cut orientations from the injection direction, are 

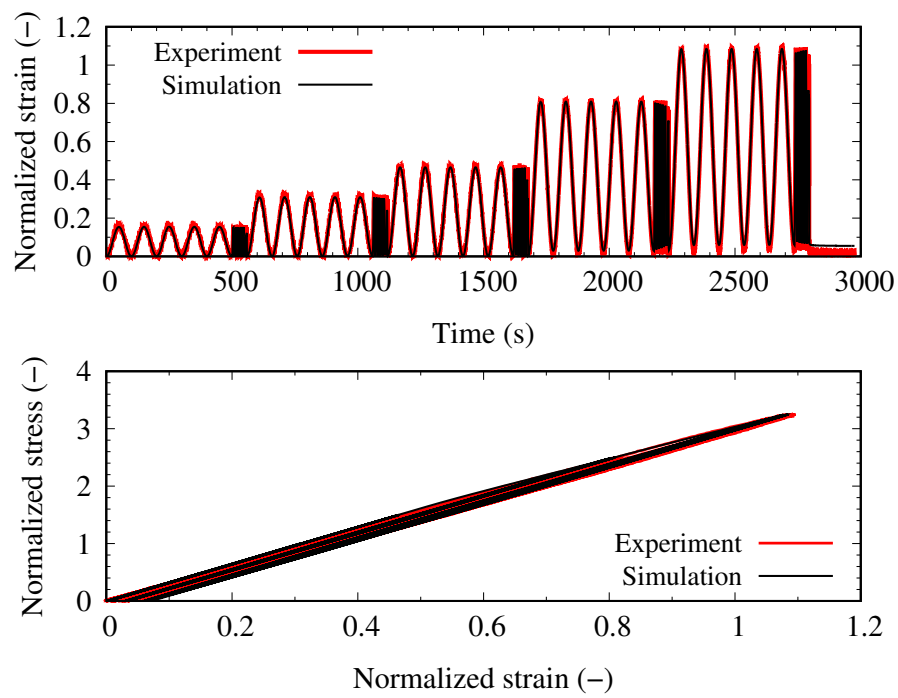

FIGURE 3 - Experimental and calculated results of a cyclic test performed on a skin sample with its gauge length along the injection direction $\left(0^{\circ}\right.$ orientation).
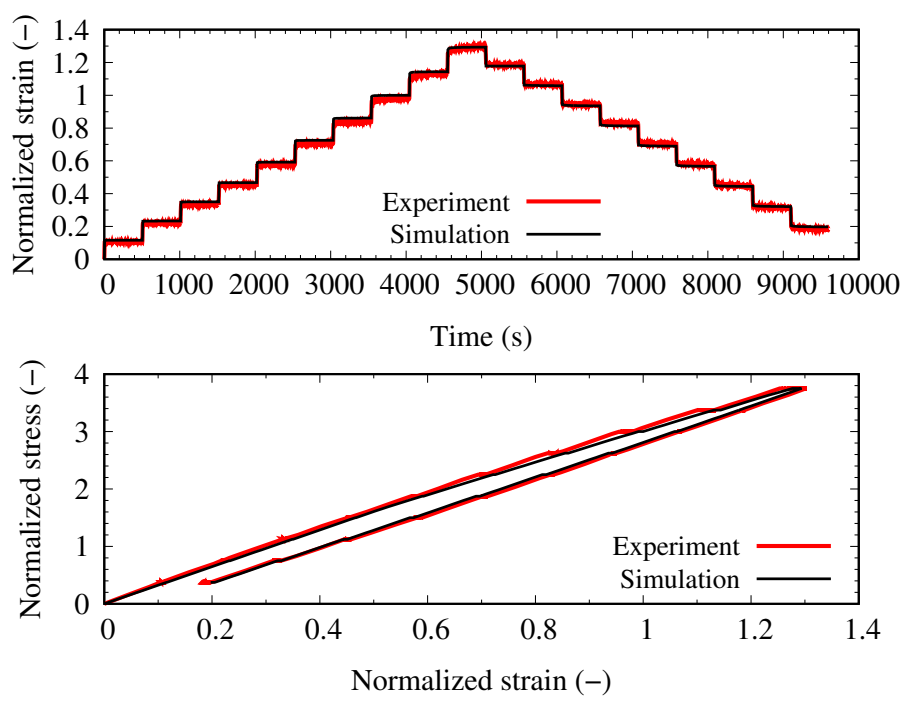

FIGURE 4 - Experimental and calculated results of an anhysteretic test performed on a skin sample with its gauge length along the injection direction $\left(0^{\circ}\right.$ orientation). 


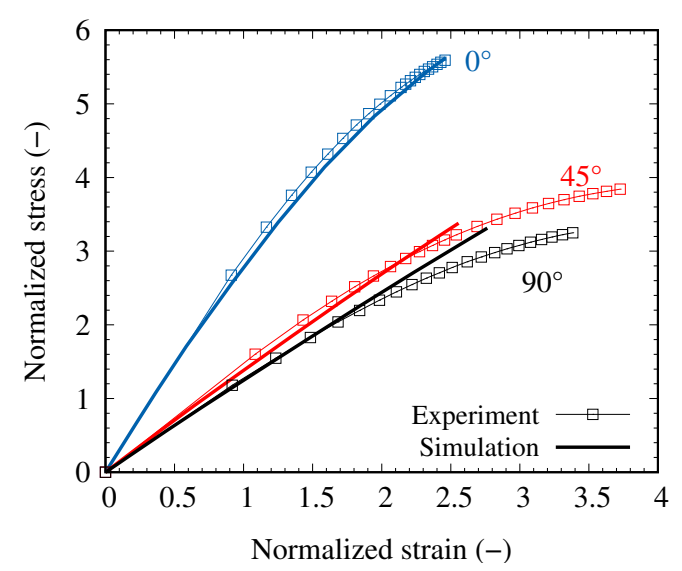

FigURE 5 - Experimental and calculated results of tensile tests performed until failure on full samples, for various loading directions from the injection direction.

correctly reproduced by the finite element simulations using the proposed model. In order to further validate the model, the experimental and calculated displacement fields at the sample surface were also compared. The relative error on the displacement fields (difference between the calculated and the measured displacements divided by the difference between the highest and the lowest displacement measured) just before failure of full samples oriented at $0^{\circ}$ and $90^{\circ}$ from the injection direction is mostly lower than $10 \%$. In addition, finite element simulations of skin samples with a hole loaded in tension along or perpendicularly to the injection direction $\left(0^{\circ}\right.$ and $90^{\circ}$ orientations, respectively), were carried out. As shown in Fig. 6, the displacement fields at the surface of the sample are well reproduced. The discrepancy between experimental and calculated results may be due to differences between the actual microstructure (fibers orientation distribution) and the microstructure used for the simulation, considered as homogeneous along the sample gauge length.

One of the main objective of the developed model regarding the application is to predict the material response when subjected to cyclic loading, in particular its intrinsic dissipation which can be related to fatigue life [1, 2]. As shown in Fig. 7, the cyclic dissipation of skin samples with a $0^{\circ}$ orientation towards from the injection direction, subjected to heat build-up tests, is correctly predicted by the model. However, intrinsic dissipation is underestimated in the case of skin samples oriented at $90^{\circ}$ from the injection direction. The tendency as a function of stress amplitude is nonetheless correctly reproduced and the dissipation for a given stress amplitude is larger for the $90^{\circ}$ orientation than for the $0^{\circ}$ orientation, which correlates well to experimental results. Anyway, the model deserves to be adjusted to get more quantitative predictions when the fiber orientation is mainly perpendicular to the loading direction. Nevertheless, cyclic dissipation of full samples, i.e. with a through-thickness gradient of microstructure, oriented at $0^{\circ}$ from the injection direction is very well reproduced by finite element simulations using the developed model (Fig. 8).

\section{Conclusions}

A wide campaign of tests with various complex mechanical loading histories, rates and amplitudes was performed at room temperature on samples with various fiber orientation distributions, cut out from injection molded plates of PEEK reinforced with 30wt.\% of short carbon fibers. The results, which may be extended to some other SFRT materials, highlighted a complex mechanical behavior : 


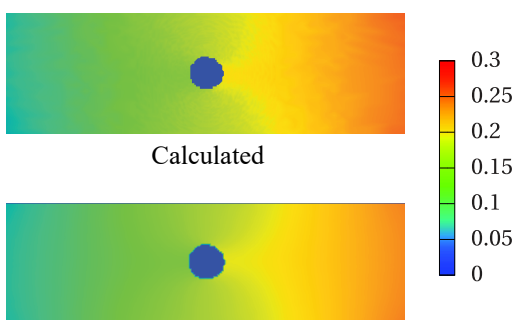

Experimental

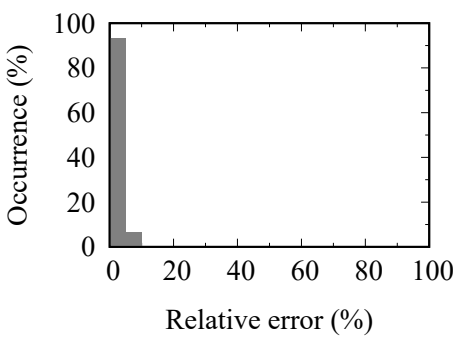

(a)

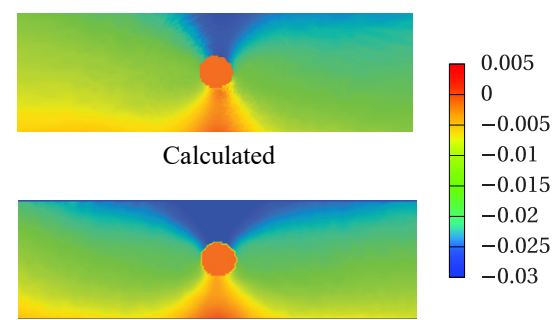

Experimental

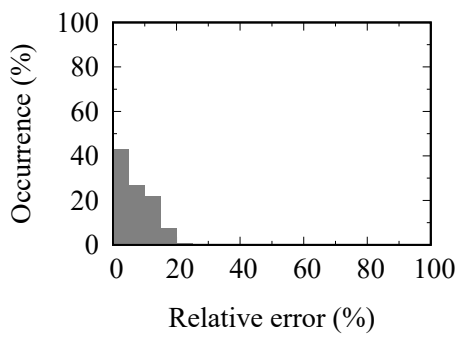

(b)

FIGURE 6 - Experimental and calculated normalized displacement fields and relative error between them, (a) along and (b) perpendicular to the (horizontal) loading direction; results obtained for a tensile test performed along the injection direction $\left(0^{\circ}\right.$ orientation) on a skin sample with a hole for a normalized stress value (calculated using the initial section of the sample without the hole) of 3.25.
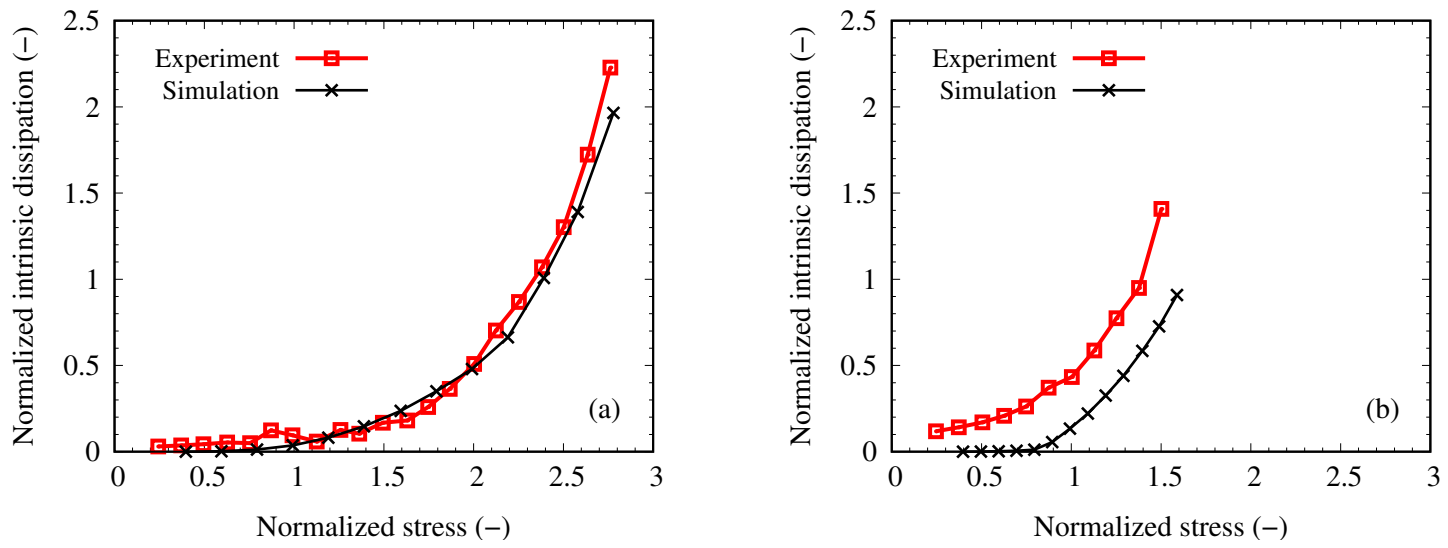

FiguRE 7 - Experimental and calculated cyclic dissipation as a function of stress amplitude for heat build-up tests performed on skin samples with their gauge length at (a) $0^{\circ}$ and (b) $90^{\circ}$ from the injection direction. 


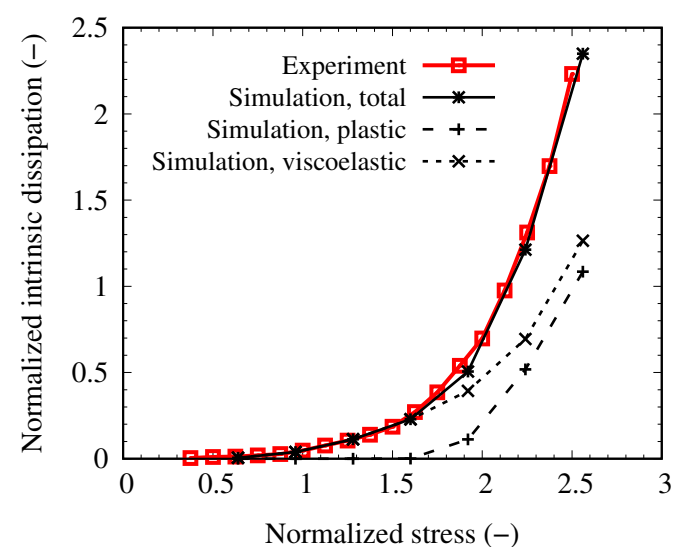

FigURE 8 - Experimental and calculated cyclic dissipation as a function of stress amplitude for heat build-up tests performed on a full sample with its gauge length at $0^{\circ}$ from the injection direction.

anisotropic elasticity, anisotropic plasticity with kinematic hardening and non-linear viscoelasticity, related to kinematic plastic hardening. A model relying on a thermodynamic framework was proposed to describe these phenomena. The model relies on a phenomenological approach and includes a limited number of parameters so that it is compatible for industrial use. A non-linear viscosity and a kinematic hardening-dependent viscoelastic stiffness is defined. Anisotropies of elasticity and plasticity are related to fiber orientation distribution so that they are naturally described. The model has been implemented into a finite element code so that the response of samples with a heterogeneous fiber orientation distribution can be simulated.

The model parameters were tuned for PEEK with 30wt.\% of short carbon fibers, following a robust and original identification procedure based on a limited number of relevant experiments performed on samples with a homogeneous and well-defined fiber orientation distribution (microstructure) and on samples with a through-thickness gradient of microstructure. Once tuned, the model was validated on the basis of results from tests not used for the identification. This validation database covers a wide range of loading history, rate and amplitude, generating homogeneous or heterogeneous loadings, and performed on samples with various homogeneous and heterogeneous fiber orientation distributions. The model accurately describes the monotonic and the cyclic mechanical behavior of the material, including dissipation under cyclic loading. The prediction of cyclic dissipation is an important output for fatigue design since dissipation can be related to fatigue properties.

\section{Références}

[1] L. Serrano, Y. Marco, V. Le Saux, G. Robert, P. Charrier, Fast prediction of the fatigue behavior of short-fiber-reinforced thermoplastics based on heat build-up measurements : application to heterogeneous cases, Continuum Mechanics and Thermodynamics, 29 (2017) 1113-1133.

[2] L. Leveuf, Y. Marco, V. Le Saux, L. Navrátil, S. Leclercq, J. Olhagaray, Fast screening of the fatigue properties of thermoplastics reinforced with short carbon fibers based on thermal measurements, Polymer Testing, 68 (2018) 19-26.

[3] L. Leveuf, L. Navrátil, V. Le Saux, Y. Marco, J. Olhagaray, S. Leclercq, Constitutive equations for the cyclic behaviour of short carbon fibre-reinforced thermoplastics and identification on a uniaxial database, Continuum Mechanics and Thermodynamics, (2018) 1-18. 
[4] A. Launay, M.H. Maitournam, Y. Marco, I. Raoult, Multiaxial fatigue models for short glass fiber reinforced polyamide - Part I : Nonlinear anisotropic constitutive behavior for cyclic response, International Journal of Fatigue, 47 (2013) 382-389.

[5] P. Santharam, T. Parenteau, P. Charrier, D. Taveau, V. Le Saux, Y. Marco, Complex fibers orientation distribution evaluation in short glass fiber-reinforced thermoplastic (PA66 GF50, MATEC Web of Conferences, 165 (2018) 22026.

[6] J.S. Cintra, C.L. Tucker, Orthotropic closure approximations for flow-induced fiber orientation, Journal of Rheology, 39 (1995) 1095-1122.

[7] A. Launay, M.H. Maitournam, Y. Marco, I. Raoult, F. Szmytka, Cyclic behaviour of short glass fibre reinforced polyamide : experimental study and constitutive equations, International Journal of Plasticity, 27 (2011) 1267-1293.

[8] I. Masquelier, Y. Marco, V. Le Saux, S. Calloch, P. Charrier, Determination of dissipated energy fields from temperature mappings on a rubber-like structural sample : Experiments and comparison to numerical simulations, Mechanics of Materials, 80 (2015) 113-123.

[9] L. Navrátil, L. Leveuf, V. Le Saux, Y. Marco, J. Olhagaray, S. Leclercq, S. Moyne, M. Le Saux, A model to describe the cyclic anisotropic mechanical behavior of short fiber-reinforced thermoplastics, To be submitted to Mechanics of Time-Dependent Materials, (2019).

[10] A. Launay, M.H. Maitournam, Y. Marco, I. Raoult, Multiaxial fatigue models for short glass fiber reinforced polyamide. Part II : fatigue life estimation, International Journal of Fatigue, 47 (2013) 390-406.

[11] B. Halphen, Q.S. Nguyen, Sur les matériaux standards généralisés, Journal de Mécanique, 14 (1975) 39-63.

[12] T. Mori, K. Tanaka, Average stress in matrix and average elastic energy of materials with misfitting inclusions, Acta Metallurgica, 21 (1973) 571-574.

[13] Y.A. Benveniste, A new approach to the application of Mori-Tanaka's theory in composite materials, Composites Science and Technology, 6 (1987) 147-157.

[14] C.H. Chen, C.H. Cheng, Effective elastic moduli of misoriented shortfiber composites, International Journal of Solids and Structures, 33 (1996) 2519-2539.

[15] G. Lielens, P. Pirotte, A. Couniot, F. Dupret, R. Keunings, Prediction of thermomechanical properties for compression moulded composites, Composites Part A : Applied Science and Manufacturing, 29 (1998) 63-70.

[16] S.G. Tandon, G.J. Weng, The effect of aspect ratio of inclusions on the elastic properties of unidirectionally aligned composites, Polymer Composites, 5 (1984) 327-333.

[17] J. Lemaitre, J.L. Chaboche, Mechanics of Solid Materials, Cambridge University Press, (1990). 